\title{
Overview of Pacific Northwest Center for Cryo-EM (PNCC): State-of-the-art electron microscopy and computational resource access free-of-charge for bioscience community
}

Irina Novikova and James Evans

Pacific Northwest National Laboratory, United States

PNCC is a national cryo-EM user facility established in 2018 to provide state-of-the-art cryo-EM capabilities for the scientific community. The facility is operated jointly by Oregon Health \& Sciences University and Pacific Northwest National Laboratory and is funded by the National Institutes of Health Common Fund. One of the main objectives of the center is the acquisition of high-resolution imaging data for users free-of-charge, which is performed by highly trained staff using the latest cryo-EM instrumentation. The facility specializes in single particle cryo-EM but also supports cryo-electron tomography (cryo-ET) projects. Over half of the research projects focus on single particle analysis of membrane protein samples due to the wealth of local expertise in this area.

The PNCC houses 5 microscopes (1 TFS Arctica and 4 Krios G3i cryo-TEMs), which are fully functional and benchmarked regularly. Each Krios microscope has been equipped with both Gatan K3 and Thermofisher Falcon III direct electron detectors. Additionally, two Krios microscopes have a Gatan energy filter and Volta phase plate. Wet lab space includes Vitrobot Mark IV and Leica GP2 plungers. Additional upgrades are planned this coming year including adding a Falcon IV direct electron detector and a Vitrojet grid preparation system. For data processing, the facility has local GPUs that provide realtime monitoring of microscope sessions using cryoSPARC Live and we also provide 1 user per project access to an allocation of 56 compute nodes on the PNNL Cascade supercomputer (each compute node is equipped with 16 CPUs and 2 NVIDIA V100 GPUs).

Interested researchers can request access to the facility and its resources via a peer reviewed proposalbased application process. There are two types of proposals accepted: general access for 5 days of microscope time and up to a 2-year duration for the project, and limited access for 1 day of microscope time (used in one time block). The proposals are scored by external reviewers and microscope access is awarded on project merit. Once awarded, PNCC staff (in particular our science points of contact) will work directly with the users to help coordinate receiving samples, microscope scheduling, sample screening, data collection conditions and even advise on image processing. In most cases the science point of contact for each project performs the actual microscope setup and collection though users can become qualified as independent users. In addition to the science point of contacts and project PIs and directors, we have a broader team of staff including user coordinators and computational scientists who assist in proposal submission and routing to data retrieval, management and assistance with high-performance computing and data processing. The facility also aids users in achieving both experimental and computing proficiency through various educational platforms: experimental and computational workshops, internships and one-on-one trainings (both on-site and virtual). Importantly, all of the above-mentioned resources are provided at no cost to the user.

In summary, PNCC aims to provide the highest quality of cryo-EM data possible, computing resources to those with no or limited such resources at their home institution and, most importantly, it aims to educate and cross-train the users to excel in their scientific independence in cryo-EM science and technology. For 
further information about the facility, User Access application process and trainings, please visit https://pncc.labworks.org.

Acknowledgement: A portion of this research was supported by NIH grant U24GM129547 and performed at the PNCC at OHSU and accessed through EMSL (grid.436923.9), a DOE Office of Science User Facility sponsored by the Office of Biological and Environmental Research
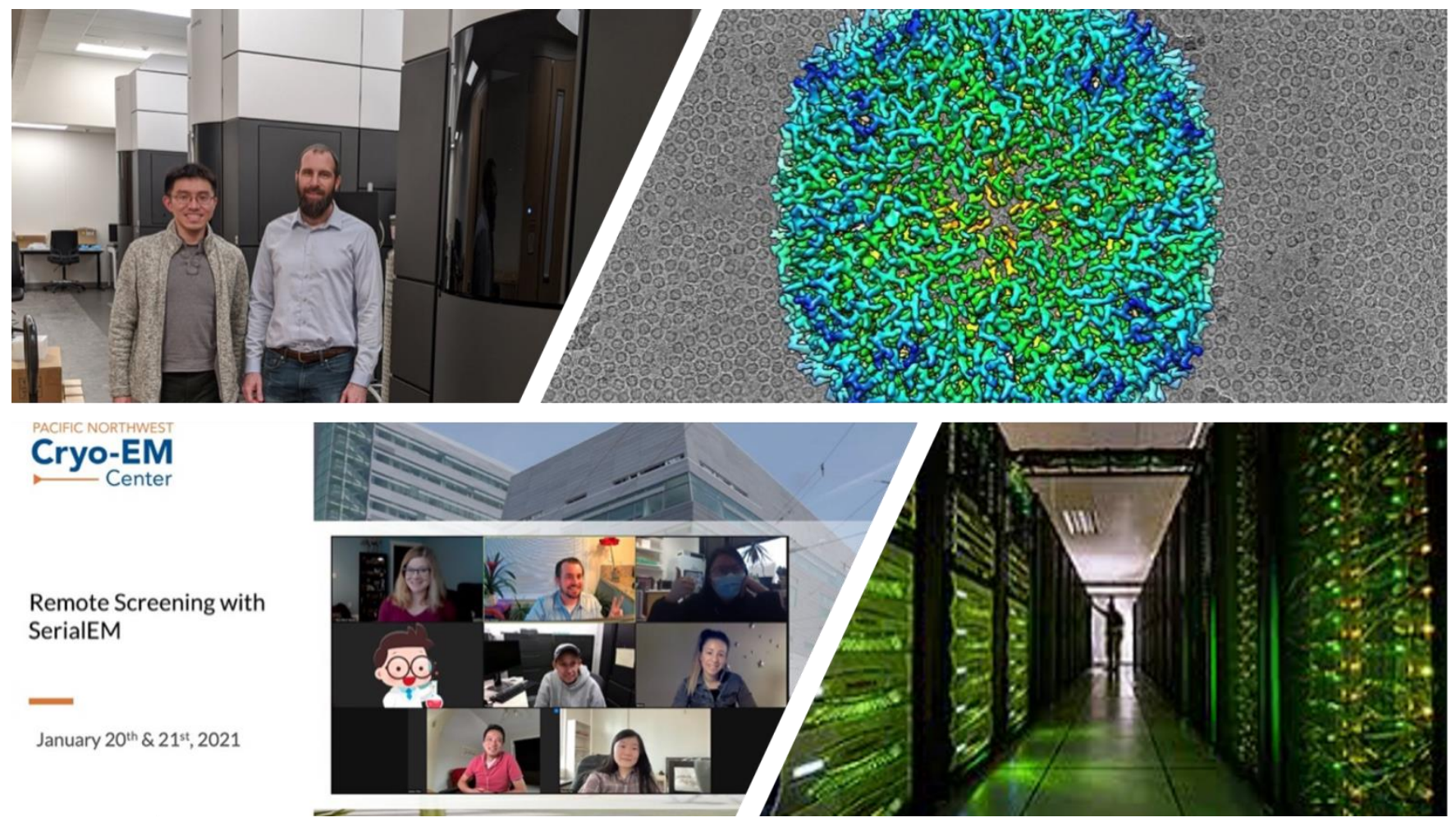

Figure 1. Montage of images associated with PNCC showing some recent benchmarking results (top right) to a view of the Cascade supercomputer users can access for image processing (bottom right) and snapshots from various remote workshops (bottom left) and one-on-one (top left) training opportunities we offer. 